\title{
EARLY RESULTS OF EXPERIMENTS TO INVESTIGATE THE FEASIBILITY OF EMPLOYING MIMO TECHNIQUES IN THE HF BAND
}

\author{
S.D. Gunashekar ${ }^{(1)}$, E.M. Warrington ${ }^{(1)}$, S. Salous ${ }^{(2)}$, S.M. Feeney ${ }^{(2)}$, H. Zhang $^{(1)}$, \\ N. Abbasi ${ }^{(1)}$, L. Bertel $^{(3)}$, D. Lemur ${ }^{(3)}$ and M.Oger ${ }^{(3)}$ \\ ${ }^{(1)}$ Department of Engineering, University of Leicester, Leicester LE1 7RH, U.K. \\ Email: sdg10@le.ac.uk,emw@le.ac.uk

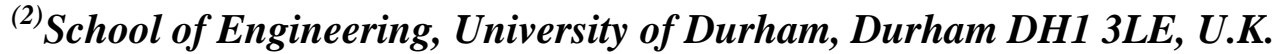

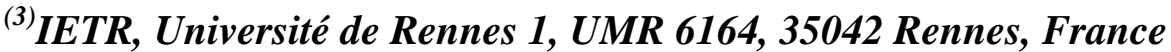

\begin{abstract}
Over the last few years, the concept of multiple input multiple output (MIMO) has become a popular area of research in the field of wireless communications with the aim of delivering increased data rates. However, to date, MIMO research has focussed primarily on communications within the VHF, UHF and SHF bands (and above). Very little experimental or modelling research has been conducted in the area of exploiting MIMO techniques within the HF radio band. This paper describes some of the preliminary experimental work that has been carried out in order to investigate the feasibility of implementing MIMO techniques within the HF band.
\end{abstract}

\section{Introduction}

An HF (3-30 MHz) propagation path through the ionosphere is prone to extensive fading as a consequence of multipath and multimode propagation [1] (the actual number of multipath components connecting a transmitter to a receiver depends on a number of factors such as the geometry of the radio link, the frequency of operation, the time of day, season, geomagnetic activity and sunspot number). As such, it would seem that the use of HF signals in a multi-element transmitter-receiver system would be an ideal candidate for the implementation of MIMO techniques. So far, however, apart from the research outlined in [2] and [3], very little experimental or modelling research has been conducted in this area. This paper describes some of the novel experimental work that has been carried out in order to investigate the feasibility of applying MIMO techniques within the HF band.

\section{Experimental Arrangement}

For the investigations presented in this paper, a purpose-built multi-channel transmitter system and multichannel receiver system was implemented. The receiver site is located in Bruntingthorpe (Leicester), UK and the transmitter site is located in Durham, UK giving a range of approximately $255 \mathrm{~km}$ (a map showing the locations of the transmitter and receiver sites is given in the left frame of Fig.1). Currently, the system has the capability of transmitting on up to four antennas and receiving on up to eight antennas. In order to investigate the effects of antenna heterogeneity at the transmitter, a variety of antennas were used in the campaigns (e.g. vertical, loop, dipole and crossed wire antennas). At the receiver, a spaced antenna array was employed so that the effect of antenna spacing could be determined. Specifically, an L-shaped array of five vertical monopole antennas (whips) was utilised in all the campaigns. As illustrated in the right frame of Fig. 1, the array consisted of a $40 \mathrm{~m}$ long N$\mathrm{S}$ arm (pointing in the general direction of Durham) and a $40 \mathrm{~m}$ long $\mathrm{E}-\mathrm{W}$ arm. Therefore, the two orthogonal arms of this configuration also facilitated the examination of any orientation-dependent propagation effects. Finally, in some of the campaigns, additional antennas (e.g. loop and crossed wires) were also utilised to introduce heterogeneity at the receiver (Fig.1, right frame). Concurrent measurements of the amplitude of CW signals received from the various transmitting antennas in Durham were made at each of the receiving antennas.

\section{Measurements and Analysis}

Between September and October 2007, a number of campaigns were performed over the DurhamBruntingthorpe radio path. The following sub-sections provide examples of two cases in which different MIMO configurations were employed. In both campaigns, data was collected over a period of approximately 1-2 hours, and the specific examples presented in each sub-section are representative of majority of the measurements. 
For the various transmit antennas, slightly different frequencies (offset by 10-20 Hz) were used. During testing, it was verified that the different frequencies exhibited correlated fading when transmitted simultaneously on a single antenna. This result confirmed that the differences in frequencies (when used on separate transmit antennas) would not contribute to any kind of de-correlation during the MIMO campaigns.
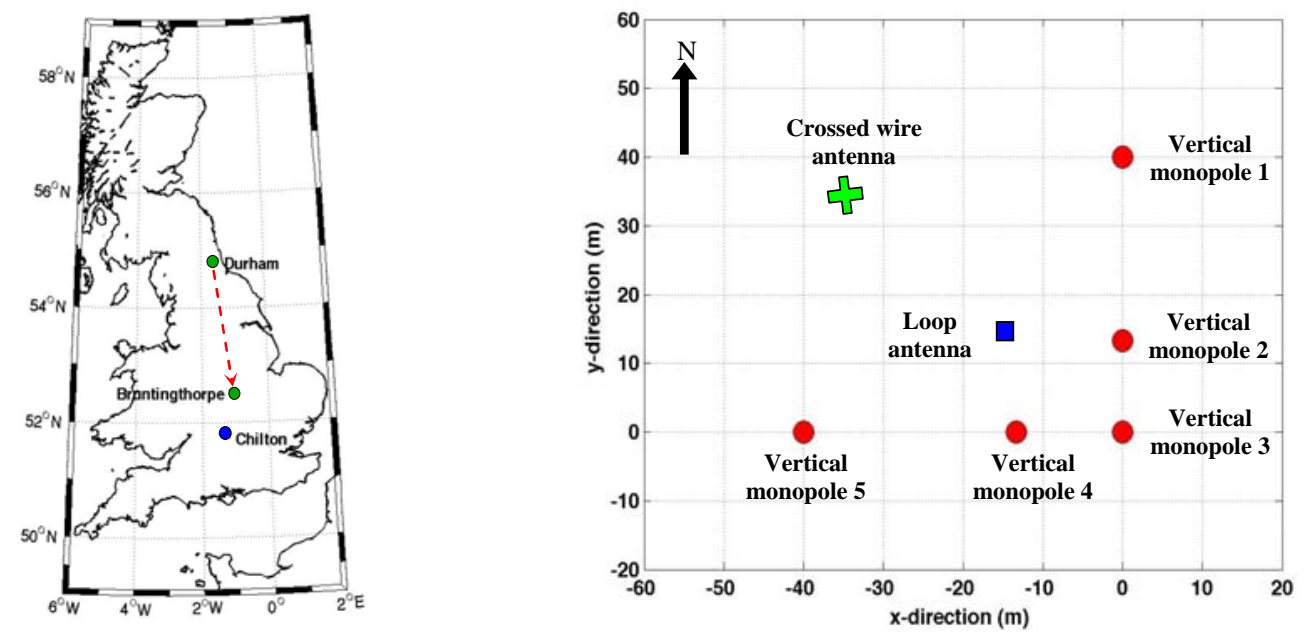

Fig.1: (Left) Map of UK depicting the locations of the transmitter and receiver sites, and the Chilton ionosonde. (Right) The relative positions of the antennas employed in the receiving antenna array in Bruntingthorpe.

\section{Example-1: 4 x 5 MIMO Configuration (17 October 2007 at 15:43 UT)}

For the measurements described in this example, a 4 x 5 MIMO configuration was utilised. For the transmit array, four different transmit antennas (vertical (TX-1): $5.255010 \mathrm{MHz}$, loop (TX-2): $5.255020 \mathrm{MHz}$, N-S crossed wire (TX-3): $5.255030 \mathrm{MHz}$ and E-W crossed wire (TX-4): $5.255040 \mathrm{MHz}$ ) were utilised, while for the receive array, the L-shaped configuration of five vertical monopoles (Fig.1, right frame) was employed.

As an example, the amplitude (linear units) pattern across two of the five monopoles of the L-shaped array for CW signals received from Durham for a period of approximately one minute (at 15:43 UT on 17 October 2007) is depicted in Fig. 2. Deep fading is observed on each of the receiving antennas for all four transmissions, indicative of the presence of a number of multipath components. Indeed, when transmission curves were superimposed on the corresponding vertical ionogram obtained from the Chilton ionosonde (location shown in Fig.1, left frame), the presence of multiple propagation modes was confirmed: in addition to the $1 F 2,2 F 2$ and 3F2 modes, both the ordinary and extraordinary modes were present. The magnitudes of the amplitude correlation coefficients between the individual transmissions at each of the receiving monopole antennas are listed in Table 1 . The values do not exceed approximately 0.85 suggesting adequate levels of de-correlation (according to [4], for a 10-element uniform linear array, the MIMO channel capacity does not degrade significantly until the inter-element correlation coefficient exceeds approximately 0.9).

The resultant correlation coefficients computed for various antenna pair spacings in this example are depicted in the left frame of Fig.3. In order to identify orientation-dependent fading effects, the data from each transmitter (indicated by the different colours) have been divided into two parts (indicated by the different symbols) depending on the orientation of the particular antenna arm: N-S arm of L-shaped array (solid circles); E-W arm of L-shaped array (solid squares). Two effects are obvious from this graph. Firstly, the correlation coefficients between the different antennas are very high. Even at the maximum antenna spacing of $40 \mathrm{~m}$, the values of correlation coefficient do not fall below 0.90. Secondly, there does not appear to be any direction-dependent behaviour, with both orthogonal arms of the L-shaped array resulting in comparable values of correlation.

The results from the correlation analysis indicate that under the prevailing multipath conditions in the ionosphere, on the one hand, the spatial distribution of antennas at the receiver is not contributing to any kind of de-correlation (and would therefore be ineffective for achieving a MIMO capacity gain). However, the spaced, heterogeneous nature of the antenna configuration (i.e. the 'antenna' diversity) employed at the transmitter is resulting in low values of correlation coefficient between the individual transmissions. 

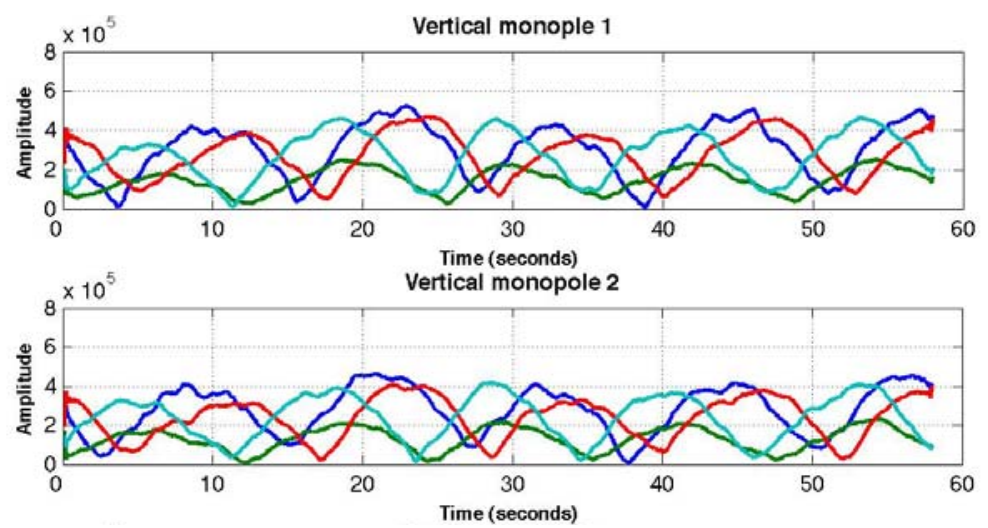

Fig. 2: Amplitude pattern observed on two channels of the 5-element L-shaped vertical monopole array at Bruntingthorpe for four CW signals received from Durham for a period of approximately one minute (at 15:43 UT on 17 October 2007) [Legend: dark blue data: vertical TX antenna (5.255010 MHz), green data: loop TX antenna (5.255020 MHz), red data: $N$-S crossed wire TX antenna (5.255030 MHz), light blue data: E-W crossed wire TX antenna (5.255040 MHz)].

\section{Example-2: 4 x 8 MIMO Configuration (02 November 2007 at 16:02 UT)}

In this example, a 4 × 8 MIMO configuration was utilised. For the transmit array, two crossed wire antennas spaced approximately $22 \mathrm{~m}$ apart were employed with the following specifications: E-W arm of crossed wire 1 (TX-1): $5.255010 \mathrm{MHz}, \mathrm{N}-\mathrm{S}$ arm of crossed wire 1 (TX-2): $5.255020 \mathrm{MHz}, \mathrm{E}-\mathrm{W}$ arm of crossed wire 2 (TX-3): 5.255030 MHz and E-W arm of crossed wire 2 (TX-4): $5.255040 \mathrm{MHz}$. The N-S arms of the two crossed wire antennas were oriented in such a way that they were pointing towards Bruntingthorpe (bearing $=173^{\circ}$ ). For the receive array, in addition to the L-shaped configuration of five vertical monopoles, a large square loop antenna (side length=1.5 m) and a crossed wire antenna were set up. The locations of the loop and crossed wire antennas relative to the L-shaped array are shown in the right frame of Fig.1. The N-S arm of the receive crossed wire antenna was pointing in the direction of Durham.

As in the previous example, the amplitude pattern across the eight receiving antennas indicated deep fading for all four transmissions. The corresponding Chilton vertical ionogram-transmission curves plot showed the presence of multiple propagation modes. The existence of a strong $E$-region in the ionosphere gave rise to many more modes ( $1 E, 2 E$ and $3 E$ ) in addition to the $1 F 2,2 F 2$ and $3 F 2$ modes (and both the ordinary and extraordinary modes) that were observed in Example 1. The magnitudes of the amplitude correlation coefficients between the individual transmissions at each of the receiving antennas have been listed in Table 1. Expectedly, the orthogonal arms of the transmit crossed wire antennas (i.e. the N-S and E-W arms) exhibit significantly lower levels of correlation compared to the parallel arms.
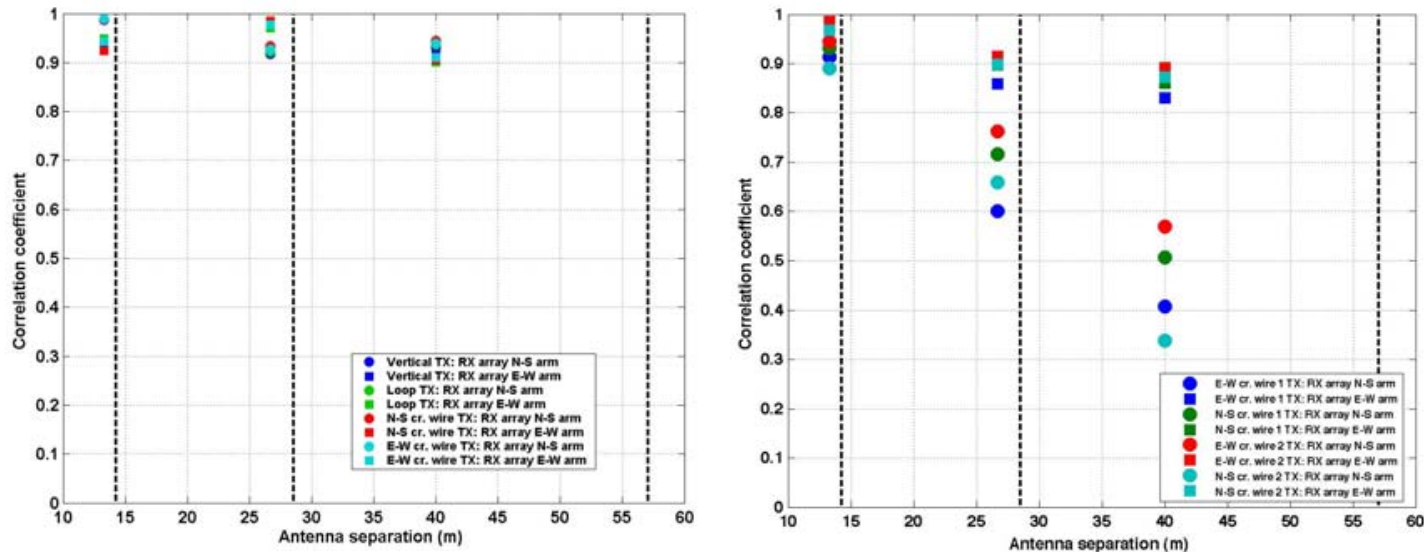

Fig. 3: The amplitude correlation coefficients computed for various antenna pair spacings of the L-shaped vertical monopole antenna array for each of the four transmissions: Example 1 (left frame) and Example 2 (right frame). The data have been divided into two parts depending on the orientation of the particular antenna arm.

The plot of amplitude correlation coefficient vs. antenna spacing (Fig.3, right frame) for the L-shaped array indicates the occurrence a strong directional effect. Compared to the E-W arm, the correlation coefficients between different pairs of antennas in the N-S arm of the L-shaped array decrease much more rapidly with antenna separation. Specifically, at the maximum antenna spacing of $40 \mathrm{~m}$, the values of correlation coefficient 
for all four transmissions drop to as low as 0.30-0.60. This behaviour could be attributed to (a) the presence of more propagation modes and/or (b) the orthogonality at the transmit end and/or (c) the fact that along the N-S arm of the L-shaped array, the incoming radio waves would have to travel comparatively different distances (therefore resulting in more de-correlation) compared to the orthogonal E-W arm. Finally, the orthogonal nature of the receive crossed wire antenna also appears to have an implication on the levels of de-correlation achieved. In particular, the correlation coefficients between the N-S and E-W arms are 0.49 (for TX-1), 0.56 (for TX-2), 0.43 (for TX-3) and 0.50 (for TX-4).

Table 1: Amplitude correlation coefficients between the various transmissions at each receiving antenna. In Example-1, $R X-$ $1=$ vertical whip $1, R X-2=$ vertical whip $2, R X-3=$ vertical whip $3, R X-4=v e r t i c a l$ whip 4 and $R X-5=v e r t i c a l$ whip 5 . In Example-2, $R X-1=$ vertical whip 1, $R X-2=$ vertical whip 2, $R X-3=$ vertical whip 3, $R X-4=$ vertical whip $4, R X-5=$ vertical whip 5, $R X-6=$ large loop antenna, $R X-7=N-S$ crossed wire and $R X-8=E-W$ crossed wire.

\begin{tabular}{|c|c|c|c|c|c|c|}
\hline & \multicolumn{7}{|c|}{ Magnitude of Amplitude Correlation Coefficients } \\
\hline Example-1 & (TX-1, TX-2) & (TX-1, TX-3) & (TX-1, TX-4) & (TX-2, TX-3) & (TX-2, TX-4) & (TX-3, TX-4) \\
\hline RX-1 & 0.157 & 0.578 & 0.295 & 0.574 & 0.852 & 0.789 \\
\hline RX-2 & 0.149 & 0.545 & 0.312 & 0.596 & 0.846 & 0.798 \\
\hline RX-3 & 0.142 & 0.565 & 0.321 & 0.586 & 0.852 & 0.807 \\
\hline RX-4 & 0.097 & 0.551 & 0.356 & 0.629 & 0.863 & 0.834 \\
\hline RX-5 & 0.206 & 0.542 & 0.265 & 0.563 & 0.843 & 0.798 \\
\hline Example-2 & (TX-1, TX-2) & $\mathbf{( T X - 1 , ~ T X - 3 ) ~}$ & (TX-1, TX-4) & (TX-2, TX-3) & (TX-2, TX-4) & (TX-3, TX-4) \\
\hline RX-1 & 0.149 & 0.628 & 0.347 & 0.067 & 0.715 & 0.081 \\
\hline RX-2 & 0.201 & 0.720 & 0.217 & 0.137 & 0.768 & 0.080 \\
\hline RX-3 & 0.154 & 0.691 & 0.220 & 0.308 & 0.735 & 0.290 \\
\hline RX-4 & 0.184 & 0.706 & 0.284 & 0.287 & 0.729 & 0.326 \\
\hline RX-5 & 0.268 & 0.805 & 0.275 & 0.259 & 0.742 & 0.252 \\
\hline RX-6 & 0.282 & 0.522 & 0.262 & 0.170 & 0.804 & 0.184 \\
\hline RX-7 & 0.137 & 0.613 & 0.070 & 0.400 & 0.805 & 0.326 \\
\hline RX-8 & 0.061 & 0.530 & 0.050 & 0.265 & 0.732 & 0.259 \\
\hline
\end{tabular}

\section{Concluding Remarks}

In order to realise increased channel capacity by means of a MIMO system, it is essential that there is sufficient de-correlation between the antenna elements at each end of the link. As shown in this analysis, at HF wavelengths, the use of homogeneous spaced arrays will require significant spacing to achieve acceptable levels of de-correlation. Marked orientation-dependent effects have also been observed. Additionally, the prevailing structure of the ionosphere and the number of multipath components that it can support has a major influence on the MIMO performance. Looking to the future, the use of co-located heterogeneous antenna arrays will be investigated, which would enable the compact implementation of a HF-MIMO system by achieving the necessary de-correlation at a single location.

\section{Acknowledgements}

The authors are grateful to the EPSRC for their financial support of this work. The collaboration between the UK and French groups in this investigation is facilitated through the EU COST-296 Action on the Mitigation of Ionospheric Effects on Radio Systems (MIERS).

\section{References}

[1] Davies, K., “Ionospheric Radio,” Peter Peregrinus Limited, UK, 1990.

[2] N.L. Brine, C.C Lim, A.D. Massie, and W. Marwood, "Capacity Estimation for the HF-MIMO Channel," Proceedings of the Sixth Symposium on Radiolocation and Direction Finding, Southwest Research Institute, San Antonio, Texas, 2006.

[3] H.J. Strangeways, "Estimation of signal correlation at spaced antennas for multi-moded ionospherically reflected signals and its effect on the capacity of SIMO and MIMO HF links,” The IET 10th International Conference on Ionospheric Radio Systems and Techniques 2006, London, UK, pp. 306-310, 2007.

[4] S.L. Loyka, "Channel capacity of MIMO architecture using the exponential correlation matrix," IEEE Communications Letters, Vol. 5, No. 9, pp 369-371, 2001. 